\title{
Diffusion about the mean drift location in a biased random walk
}

\author{
Edward A. Codling, ${ }^{1,3}$ Rachel N. Bearon, ${ }^{2}$ and Graeme J. Thorn ${ }^{1}$ \\ ${ }^{1}$ Departments of Mathematical Sciences and Biological Sciences, University of Essex, Wivenhoe Park, \\ Colchester CO4 3 SO United Kingdom \\ ${ }^{2}$ Department of Mathematical Sciences, Mathematics and Oceanography Building, University of Liverpool, \\ Peach Street, Liverpool L697ZL United Kingdom
}

\begin{abstract}
Random walks are used to model movement in a wide variety of contexts: from the movement of cells undergoing chemotaxis to the migration of animals. In a twodimensional biased random walk, the diffusion about the mean drift position is entirely dependent on the moments of the angular distribution used to determine the movement direction at each step. Here we consider biased random walks using several different angular distributions and derive expressions for the diffusion coefficients in each direction based on either a fixed or variable movement speed, and we use these to generate a probability density function for the long-time spatial distribution. We demonstrate how diffusion is typically anisotropic around the mean drift position and illustrate these theoretical results using computer simulations. We relate these results to earlier studies of swimming microorganisms and explain how the results can be generalized to other types of animal movement.
\end{abstract}

Key words: angular distribution; animal movement; biased random walk; diffusion; dispersal; driftdiffusion.

\section{INTRODUCTION}

Random walk models are frequently used to model biological movement such as the dispersal of cells, microorganisms, and animals (Codling et al. 2008). At the simplest level, a random walk consists of a series of steps (possibly of different size) in randomly chosen directions. A global directional bias can be introduced into the random walk by making the probability of moving in a certain direction greater. Paths that contain a consistent bias in a preferred direction or toward a given target are termed biased random walks (BRW), or biased and correlated random walks (BCRW) if forward persistence is also observed (Patlak 1953, Benhamou 2006, Codling et al. 2008; forward persistence is a shortterm localized bias corresponding to the natural tendency for most animals to continue moving in the same direction). The global bias may be due to fixed external environmental factors (e.g., bottom heavy microorganisms moving upward under gyrotaxis; Hill and Häder 1997), to spatially varying factors, such as chemical gradients (Alt 1980, Berg 1983, Othmer et al. 1988), to mean-reversion mechanisms, such as movement within a home range, or to choice of direction by individuals at each step (Benhamou 2003). Furthermore,

Manuscript received 24 September 2009; revised 25 January 2010; accepted 18 February 2010. Corresponding Editor: S. J. Schreiber.

${ }^{3}$ E-mail: ecodling@essex.ac.uk the target direction and strength of bias are not necessarily fixed over the whole path and may vary with location and time (e.g., fish larvae aiming for a reef in Codling et al. 2004). If every individual has the same preferred direction over all space and time it is possible to quantify the direction, functional form and magnitude of the introduced bias (Benhamou and Bovet 1992, Hill and Häder 1997, Codling and Hill 2005a). However, due to the localized directional bias (forward persistence) in typical (unbiased) animal movement paths, it is a non-trivial problem to distinguish between unbiased persistent movement paths and biased movement paths when individuals have different target directions and observed paths are short (Benhamou 2006, Coscoy et al. 2007).

Typically, when considering a theoretical model of directed animal movement, we are interested in the spatial distribution across all time scales (Okubo and Levin 2001), i.e., ideally we aim to model a BCRW where both short-term forward persistence and longterm global bias effects are present (Patlak 1953). However, if the effects of short-term forward persistence are not important and we are interested in the "longtime" spatial distribution, then the movement process can be modeled as a simple BRW, making analysis easier. If the BRW has a fixed global bias such that every individual has the same preferred direction over all space and time then, at the long-time limit, the governing equation for such a process is the drift-diffusion (or 
advection-diffusion) equation. This has a bivariate Gaussian distribution as the solution (Cheung et al. 2008, Codling et al. 2008), which facilitates an analytic description of the limiting spatial distribution.

In this paper, we consider a simple two-dimensional BRW and demonstrate how, in the long-time limit, the probability density function for the distribution in space, $p(x, y, t)$, and the associated diffusion coefficients, $D_{x}$ and $D_{y}$, are dependent on the moments of the angular distribution used to determine the direction of movement at each step of the random walk. We relate this result to earlier studies on swimming microorganisms and derive analytic expressions for the diffusion coefficients (and hence the predicted spatial distribution) for four standard angular distributions: von Mises, truncated normal, wrapped normal, and wrapped Cauchy. We demonstrate how including a variable speed can significantly affect the diffusion and overall spatial distribution. The theoretical results derived have subsequently been compared to the results of simulated BRWs and there is a good match between theory and simulation. We discuss these results in the context of modeling and analysis of animal movement paths.

\section{Theoretical Model}

\section{A two-dimensional biased random walk}

We assume that our walker is moving in an unrestricted two-dimensional Euclidean space where there is a global preferred direction of movement (a "point at infinity" such that the direction is the same for every individual at all spatial positions; Benhamou 2006). The walker starts at the origin facing a random direction. Subsequently, at each step of the random walk, $\tau$, the walker moves a distance, $\tau \times s$, where the speed $s$ is randomly drawn from a specified probability distribution with mean $\bar{\delta}$ and variance $\sigma_{\delta}^{2} \times(\bar{\delta})^{2}$. For the purposes of the theoretical model, any probability distribution can be used for the speed as long as the variance is finite. Experimental studies on swimming microorganisms have shown movement speeds are typically either exponentially distributed (e.g., Hill and Häder 1997, Codling and Hill 2005a) or normally distributed (truncated Gaussian; e.g., Bearon and Grunbaum 2008). A truncated power law (which has a finite variance) could also be used, but note that a nontruncated power law distribution with $\mu<3$ as found in some Lévy flight movement models would not fit into this approach as the variance is infinite (Newman 2005). At the end of each movement step, the random walker reorients to move in a new global direction $\theta$, which is randomly drawn from an angular distribution $f(\theta)$, where $\int_{-\pi}^{\pi} f(\theta) d \theta$, and $f(\theta)$ is time and space independent. Without loss of generality, we assume that the mean angle of $f(\theta)$ is given by $\theta_{0}=0$, and that this corresponds to the positive $x$-axis in our domain. Hence, this is a position jump process (Othmer et al. 1988) and forward persistence is not explicitly accounted for in the model. This model is equivalent to the allothetic random walk model described by Cheung et al. (2007, 2008), who considered a problem relating to animal navigation, although the general BRW model described is not restricted to this context and can also be used to account for external processes such as gyrotaxis (Hill and Häder 1997) that may influence the movement direction.

If we make the further simplifying assumption that $f(\theta)$ is symmetric about $\theta_{0}$ then the mean displacements in the $x$ - (global bias, $\left.\theta_{0}\right)$ and $y$ - (non-bias, $\theta_{0}+\pi / 2$ ) directions at each step are given respectively by the following (Marsh and Jones 1988, Cheung et al. 2007, 2008, Codling et al. 2008):

$$
\mathrm{E}(X)=\mu_{X}=\bar{\delta} \int_{-\pi}^{\pi} \cos \theta f(\theta) d \theta=\bar{\delta} \rho
$$

and

$$
\mathrm{E}(Y)=\mu_{Y}=\bar{\delta} \int_{-\pi}^{\pi} \sin \theta f(\theta) d \theta=0
$$

where $0 \leq \rho \leq 1$ is the mean vector length of the angular distribution $f(\theta)$.

Similarly, using standard results for the moments of angular distributions (Mardia and Jupp 1999), the variances of the displacements are given by the following (Cheung et al. 2007, 2008):

$$
\begin{aligned}
& \operatorname{Var}(X)=(\bar{\delta})^{2}\left[\left(1+\sigma_{\delta}^{2}\right)\left(\int_{-\pi}^{\pi} \cos ^{2} \theta f(\theta) d \theta\right)-\rho^{2}\right] \\
& \operatorname{Var}(Y)=(\bar{\delta})^{2}\left[\left(1+\sigma_{\delta}^{2}\right)\left(\int_{-\pi}^{\pi} \sin ^{2} \theta f(\theta) d \theta\right)\right]
\end{aligned}
$$

which arise from the standard fact that $\operatorname{Var}(X)=\mathrm{E}\left(X^{2}\right)$ $-(\mathrm{E}(X))^{2}$ and $\operatorname{Var}(Y)=\mathrm{E}\left(Y^{2}\right)-(\mathrm{E}(Y))^{2}$. The expressions in Eqs. 2 and 3 are valid for all steps of the random walk (Cheung et al. 2007, 2008), even though at short times the spatial distribution around the mean position is non-Gaussian. However, after a large enough number of steps the central limit theorem will apply and the population spread around the mean position will be approximately diffusive (a Gaussian spatial distribution). As we discuss later, the number of steps required before this diffusion approximation applies is inversely proportional to $\rho$; see Hillen (2002) for further discussion about diffusive limits of random walks.

In this long-time limit, the probability density function for the spatial distribution at time $t$ is as follows (Cheung et al. 2008, Codling et al. 2008):

$$
p(x, y, t)=\frac{1}{4 \pi \sqrt{D_{x} D_{y}} t} \exp \left(-\frac{(x-U t)^{2}}{4 D_{x} t}-\frac{y^{2}}{4 D_{y} t}\right)
$$


TABLE 1. Standard angular distributions considered in this study, their parameters, and mean vector lengths.

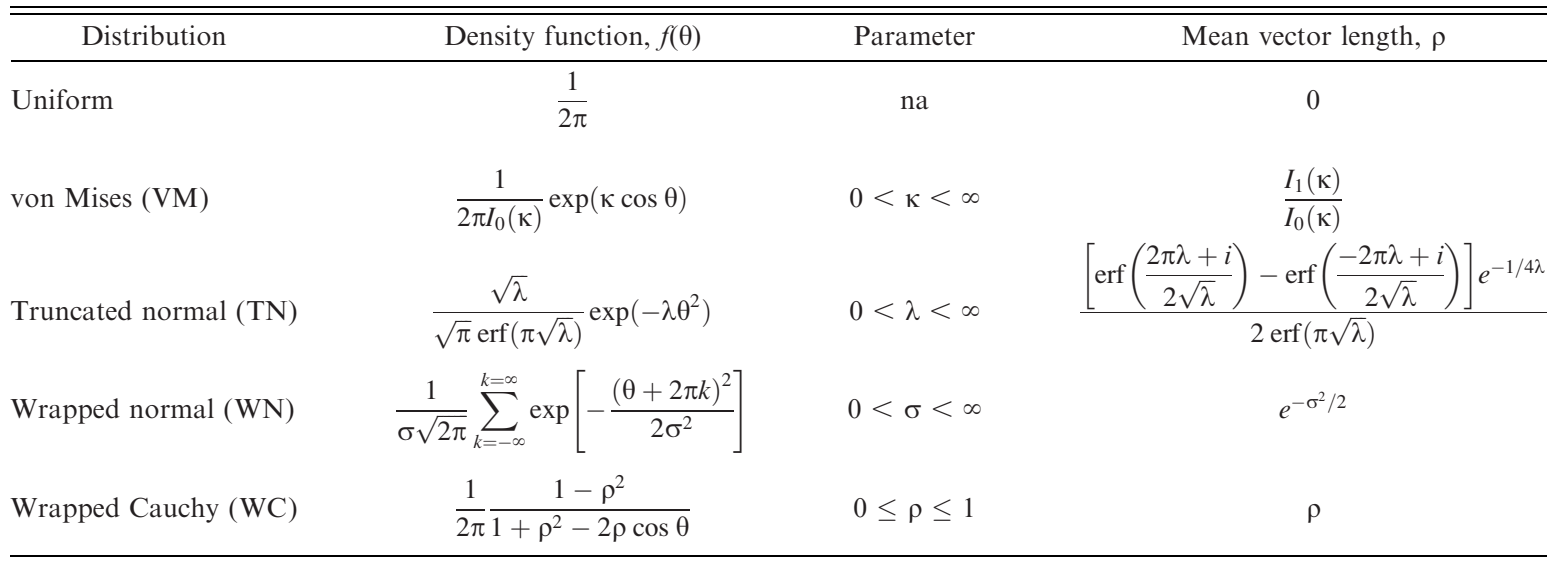

Notes: The random variable giving the choice of direction at each step of the biased random walk (BRW) is $\theta$. In the VM distribution, $I_{n}(\kappa)$ is the modified Bessel function of first kind and order $n$, where $\kappa$ is the concentration parameter. In the TN distribution, erf is the standard error function which can be calculated numerically (where $i=\sqrt{-1}$ ); $\lambda$ is the parameter that controls the spread of the distribution. In the WN distribution, $\sigma$ is the angular variance. The WC is defined in terms of the mean vector length, $\rho$. The abbreviation na stands for "not applicable."

where the drift, $U$, and diffusion, $D_{x}$ and $D_{y}$, are defined as

$$
\begin{aligned}
U & =\frac{\bar{\delta}}{\tau} \rho \\
D_{x} & =\frac{(\bar{\delta})^{2}}{4 \tau}\left[\left(1+\sigma_{\delta}^{2}\right)\left(\int_{-\pi}^{\pi}(1+\cos 2 \theta) f(\theta) d \theta\right)-2 \rho^{2}\right]
\end{aligned}
$$

$$
D_{y}=\frac{(\bar{\delta})^{2}}{4 \tau}\left(1+\sigma_{\delta}^{2}\right)\left(\int_{-\pi}^{\pi}(1-\cos 2 \theta) f(\theta) d \theta\right)
$$

In the case of a fixed speed of movement (such that $\sigma_{\delta}^{2}=$ 0 ), these results can also be derived using a difference equation and a transformation of coordinates relative to the mean drift location but we omit the details here; the approach is similar to the examples given in Codling et al. (2008). In our derivation, we assume our random walker is moving with a fixed or variable speed and turns once per time unit and our solutions are given in terms of the total time, $t$. The same expressions can be derived for a random walk where the walker moves with a constant speed for a variable distance before turning if one considers the number of steps moved, $n$, rather than the total time taken.

\section{Biased random walks for different angular distributions}

Given the results of the previous section, it is of interest to determine the long-time steady-state spatial distribution and relative spatial diffusivity for biased random walks that use different angular distributions, $f(\theta)$. Table 1 gives the probability density functions (pdf) for several relevant angular distributions and these are illustrated for both low (Fig. 1a) and high (Fig. 1b) values of the mean vector length, $\rho$. We are particularly interested in the von Mises (VM) and truncated normal (TN) distributions because they arise as the long-time steady-state movement direction distributions for experimentally observed reorientation models in the random walk on a circle for swimming microorganisms (Hill and Häder 1997, Codling and Hill 2005a). Meanwhile, the wrapped normal (WN) is a commonly occurring angular distribution in movement models, mainly because it is easy to simulate and is analogous to the standard linear normal distribution (Mardia and Jupp 1999). The wrapped Cauchy (WC) takes a slightly different form from the other distributions for large values of $\rho$, being more highly peaked with fatter tails (Mardia and Jupp 1999; and Fig. 1b). Nevertheless, the WC is often used in models of animal movement, mainly because it is easily parameterized using the mean vector length $\rho$. The uniform distribution (which can be considered a special case of any of the other angular distributions where $\rho=$ 0 ) corresponds to random unbiased movement equivalent to Brownian motion (Codling et al. 2008) and is included as a null model.

From the expressions for $\rho$ in Table 1 it is easy to find the predicted drift, $U$, for each distribution using Eq. 5. Table 2 gives the full mathematical expressions for the diffusion parameters, $D_{x}$ and $D_{y}$, (Eqs. 6-7) with either a fixed $\left(\bar{\delta}=\delta\right.$ and $\left.\sigma_{\delta}^{2}=0\right)$ or variable speed, assuming the spatial distribution is Gaussian about the mean position (the system is in the long-time diffusive steady-state). In the variable speed case, we consider in detail the cases with VM and WC angular distributions and when $\sigma_{\delta}^{2}=1$ which corresponds directly to an exponential speed distribution (Hill and Häder 1997, Codling and Hill 2005a), but can also be considered as a particular special 

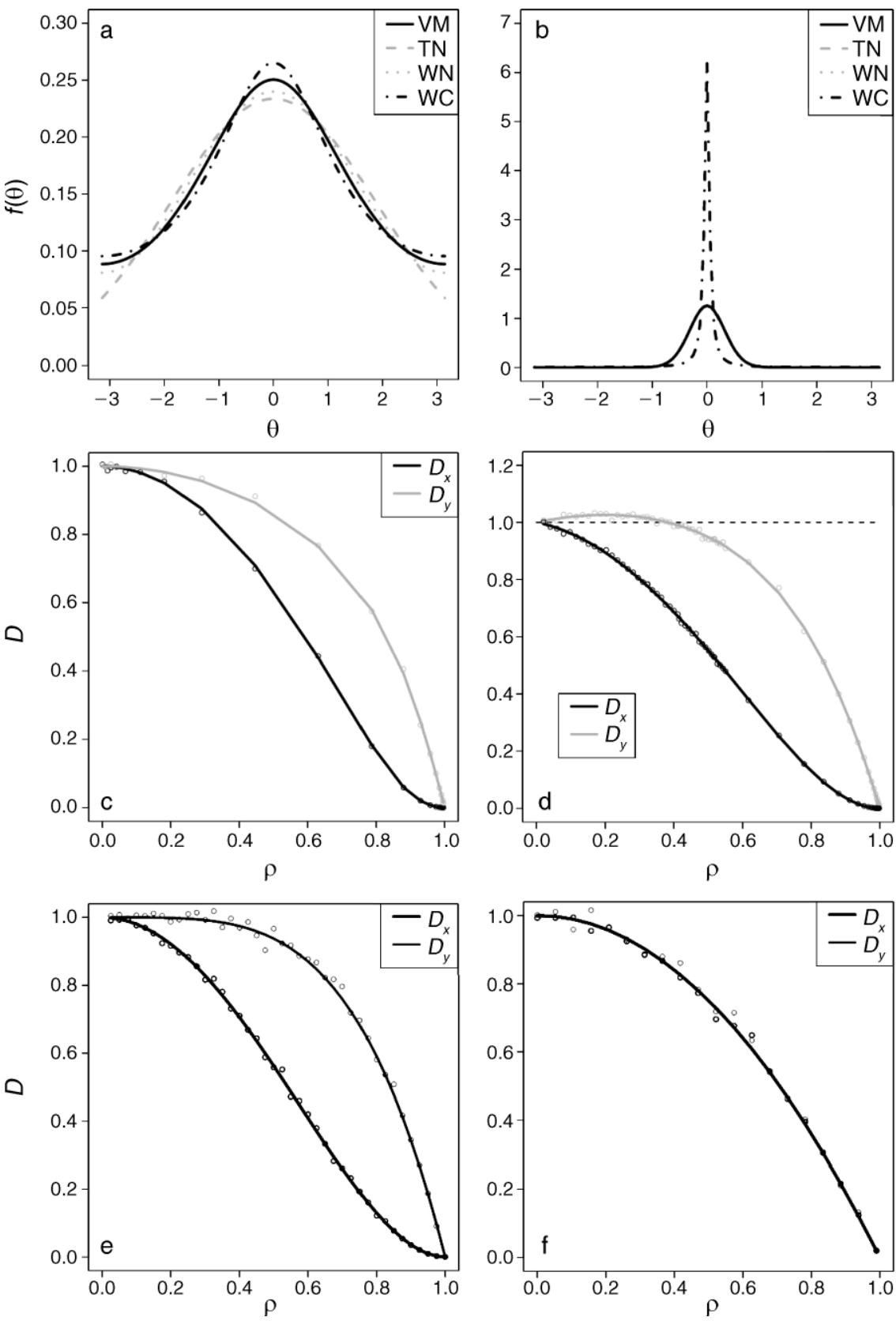

FIG. 1. (a-b) Example angular distributions $f(\theta)$ with (a) mean vector length $\rho=0.25$ and (b) $\rho=0.95$. (c-f) Comparing simulated biased random walks (BRWs) to long-time theoretical predictions for (c) von Mises (VM), (d) truncated normal (TN), (e) wrapped normal (WN), and (f) wrapped Cauchy (WC) angular distributions. Diffusion coefficients in the drift, $D_{x}$, and nondrift, $D_{y}$, directions are rescaled by a factor of $4 \tau / \delta^{2}$ so that $0 \leq D_{x}, D_{y} \leq 1$. Theoretical results are marked as black $\left(D_{x}\right)$ or gray $\left(D_{y}\right)$ solid lines; simulation results are marked as points. In these scenarios, the speed of movement is constant.

case of a Gaussian (Bearon and Grunbaum 2008) or any other finite variance speed distribution.

\section{Simulation Results}

To validate our theoretical predictions, simulations of BRW were run and compared to Eqs. 5-7 as described in the Appendix. The behavior of the biased random walk with fixed speed and VM distribution (Fig. 1c) is qualitatively similar to the TN (Fig. 1d) and WN distributions (Fig. 1e), as might be expected given the similarity of the distributions (Fig. 1a, b). In particular, it is clear that $D_{x}<D_{y}$ (Fig. 1c-e), i.e., diffusion about 
TABLE 2. Theoretical expressions for the diffusion coefficients, $D_{x}$ and $D_{y}$, calculated for the given angular distributions from Eqs. 6 and 7.

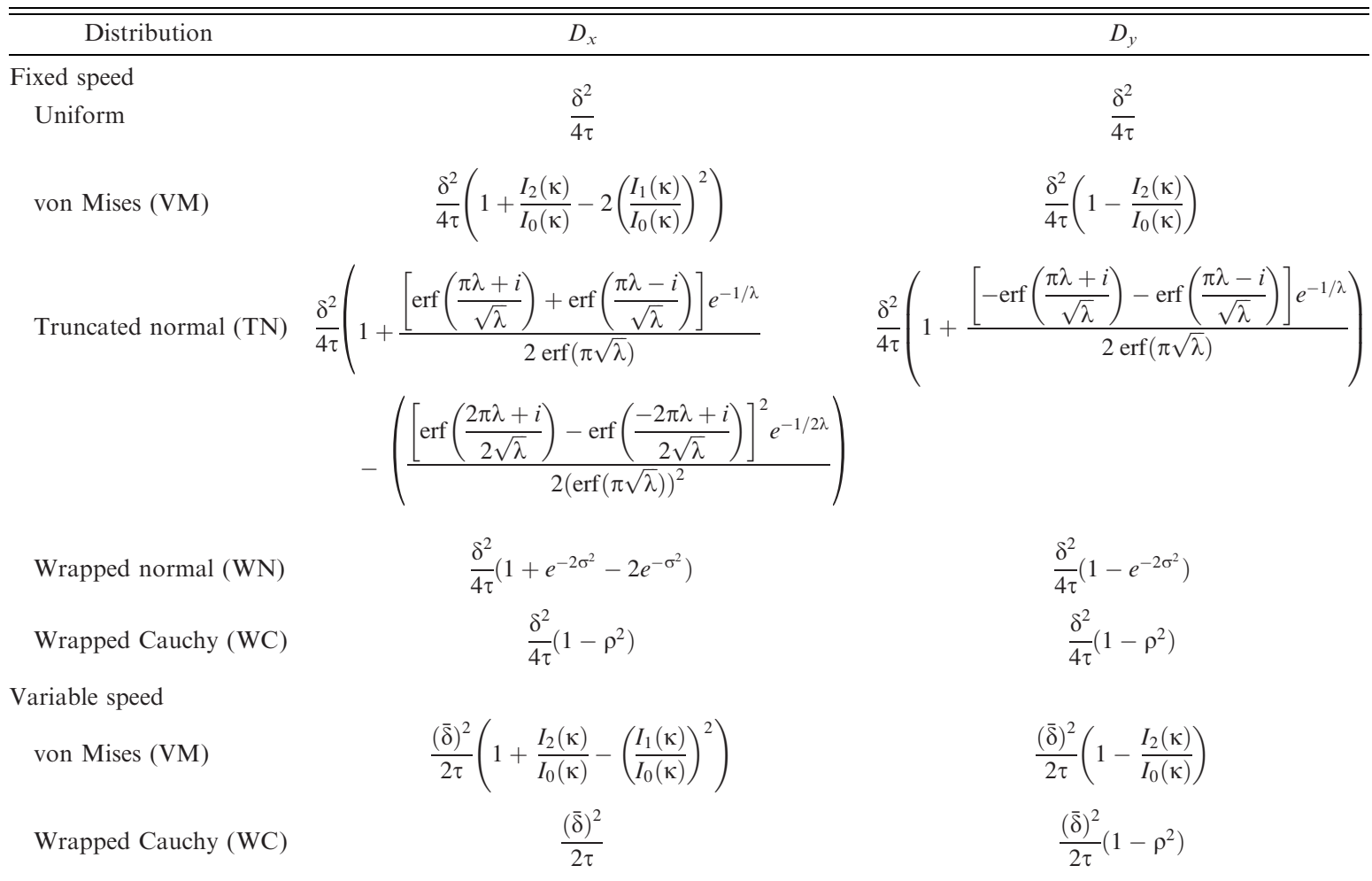

Notes: The distance moved in each time step $\tau$ is $\delta$. In the VM distribution, $I_{n}(\kappa)$ is the modified Bessel function of first kind and order $n$. In the TN distribution, erf is the standard error function which can be calculated numerically. In the variable speed case, we assume that the speed at each step is drawn from an exponential distribution with mean speed $\bar{\delta}$ and variance $\sigma_{\delta}^{2}=1$.

the mean drift location is anisotropic. Fig. 2a, e shows a good fit between simulated BRWs and the theoretical probability density function, $p(x, y, t)$ given in Eq. 4 , where the simulations have been run for sufficient time such that the spatial distribution is Gaussian. Note that the time required to reach a Gaussian distribution is itself dependent on the level of bias in the random walk, and for large values of $\rho$ a longer time is required for the theoretical solution to be valid (Fig. 2c). At shorter times, there is a clear skew in the distribution of simulated random walkers in the direction of the mean drift and the spatial distribution is not Gaussian. Similar matches between $p(x, y, t)$ and simulation results are obtained for the $\mathrm{TN}$ and $\mathrm{WN}$ distributions and are omitted. Interestingly, with the TN distribution (Fig. $1 c), D_{y}$ actually starts to increase as $\rho$ increases. Using the rescaling used in Fig. $1\left(4 \tau / \delta^{2}\right)$, simple numerical calculations show that the maximum value is given by $D_{y} \approx 1.026$ (three decimal places) for $\rho \approx 0.200$ (three decimal places). For $\rho>0.2, D_{y}$ starts to decrease and approaches zero quite steeply as $\rho \rightarrow 1$. These theoretical predictions are matched by the simulation results. Note, however, that for all values of $\rho$ we have $D_{x}+D_{y}<2$, so that the total diffusion is always less than the equivalent diffusion in an unbiased random walk (where we would expect $D_{x}=D_{y}=1$ ). For the WC reorientation model, diffusion about the mean location is the same in both the drift and non-drift directions, $D_{x}$ $=D_{y}$ (Fig. 1f). This is qualitatively different behavior to that found with the other three angular distributions considered and is likely to arise as a consequence of the different shape of the WC distribution (Fig. 1b). As with the VM distribution, a good match is obtained between $p(x, y, t)$ and simulation results (Fig. 2b, f), assuming the simulations are run for sufficient time such that the spatial distribution is Gaussian.

With the inclusion of a variable speed (specifically, where the speed at each step is drawn from an exponential distribution with $\sigma_{\delta}^{2}=1$ ), the VM model gives spatial diffusion that is as much as twice as large as the fixed speed case (Fig. 3a; even though the mean speed is the same for both models). This is unsurprising as a variable speed increases the spatial variance. From Fig. 3a, it is interesting to note that $D_{x}$ initially decreases as $\rho$ increases, but then increases and (given the rescaling $4 \tau /(\bar{\delta})^{2}$ used in the figures) $D_{x} \rightarrow 2$ as $\rho \rightarrow 1$. In contrast to the fixed speed case, $D_{x}>D_{y}$, although $D_{y}$ does decrease as $\rho$ increases (Fig. 3a). In the extreme case of $\rho$ 

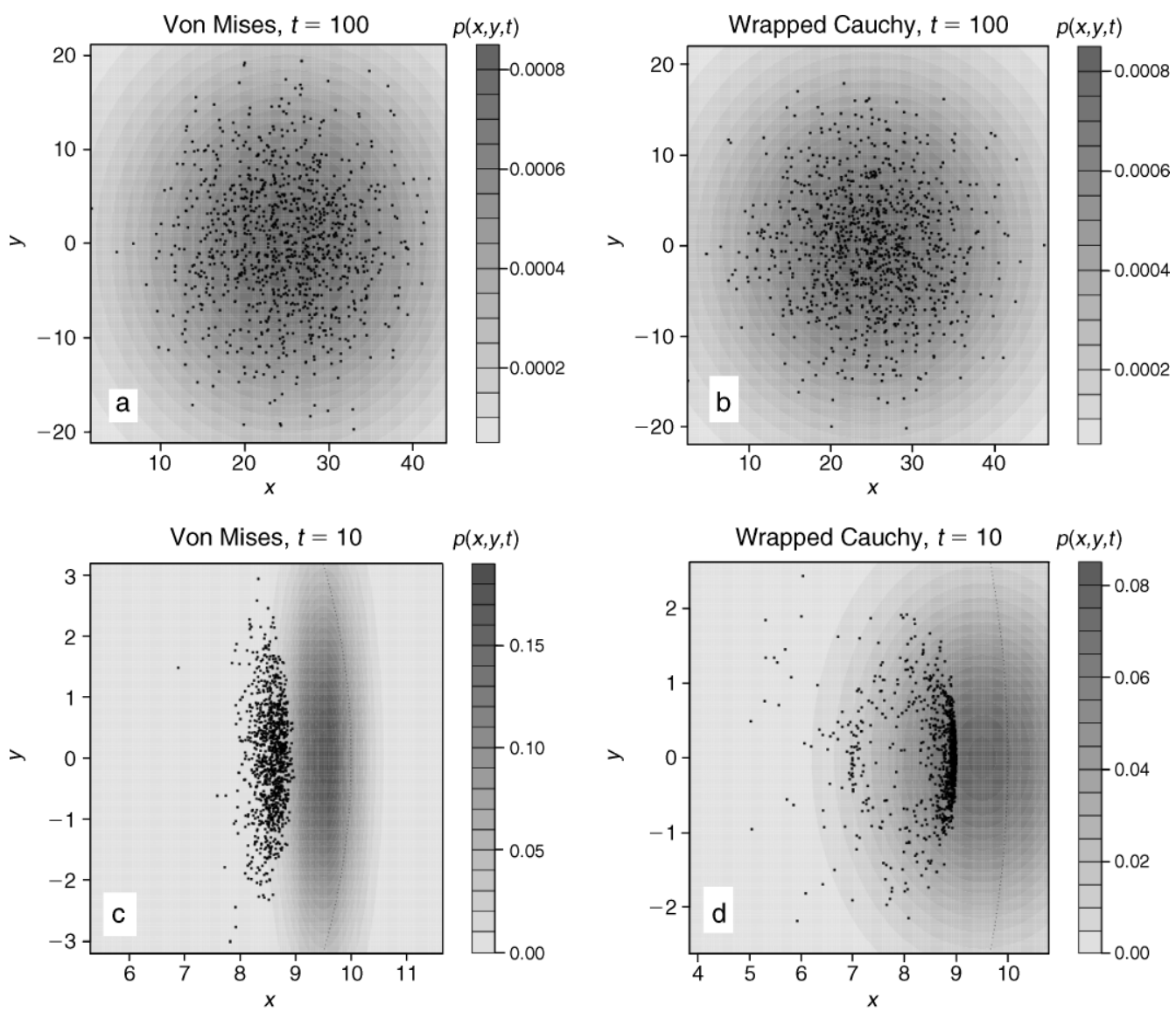

$p(x, y, t)$
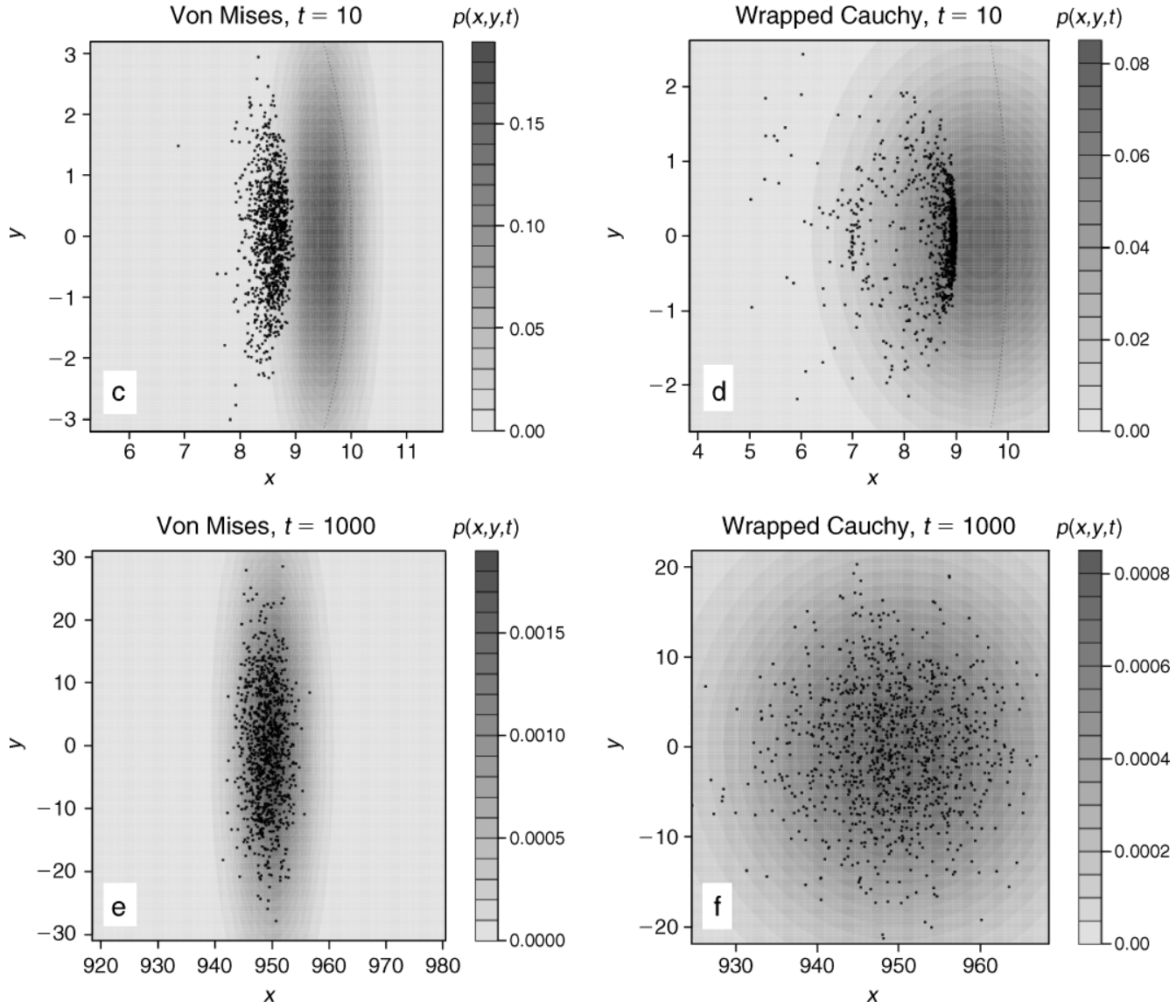

FIG. 2. Theoretical probability density plots calculated from Eq. 4 for (a, c, e) von Mises and (b, d, f) wrapped Cauchy angular distributions for $(\mathrm{a}, \mathrm{b}) t=100$ and $\rho=0.25$, (c, d) $t=10$ and $\rho=0.95$, and $(\mathrm{e}, \mathrm{f}) t=1000$ and $\rho=0.95$, where $t$ is the number of time steps and $\rho$ is the mean vector length. The end points of 1000 simulated BRWs are marked as points. In these scenarios, the speed of movement is constant.

$\rightarrow 1$ such that movement is in the drift $(x)$ direction only, it is unsurprising that $D_{y} \rightarrow 0$. However, in this situation, as the speed is variable the position of each individual along the drift direction is itself variable, leading to higher diffusion. Results with the TN and
WN angular distributions are qualitatively similar and are omitted. In contrast, it is interesting to note that (given the rescaling $\left.4 \tau /(\bar{\delta})^{2}\right)$, the WC distribution with variable speed has $D_{x}=2$ for all $\rho . D_{y}$ is now twice as large as with a fixed speed for all values of $\rho$, but this 

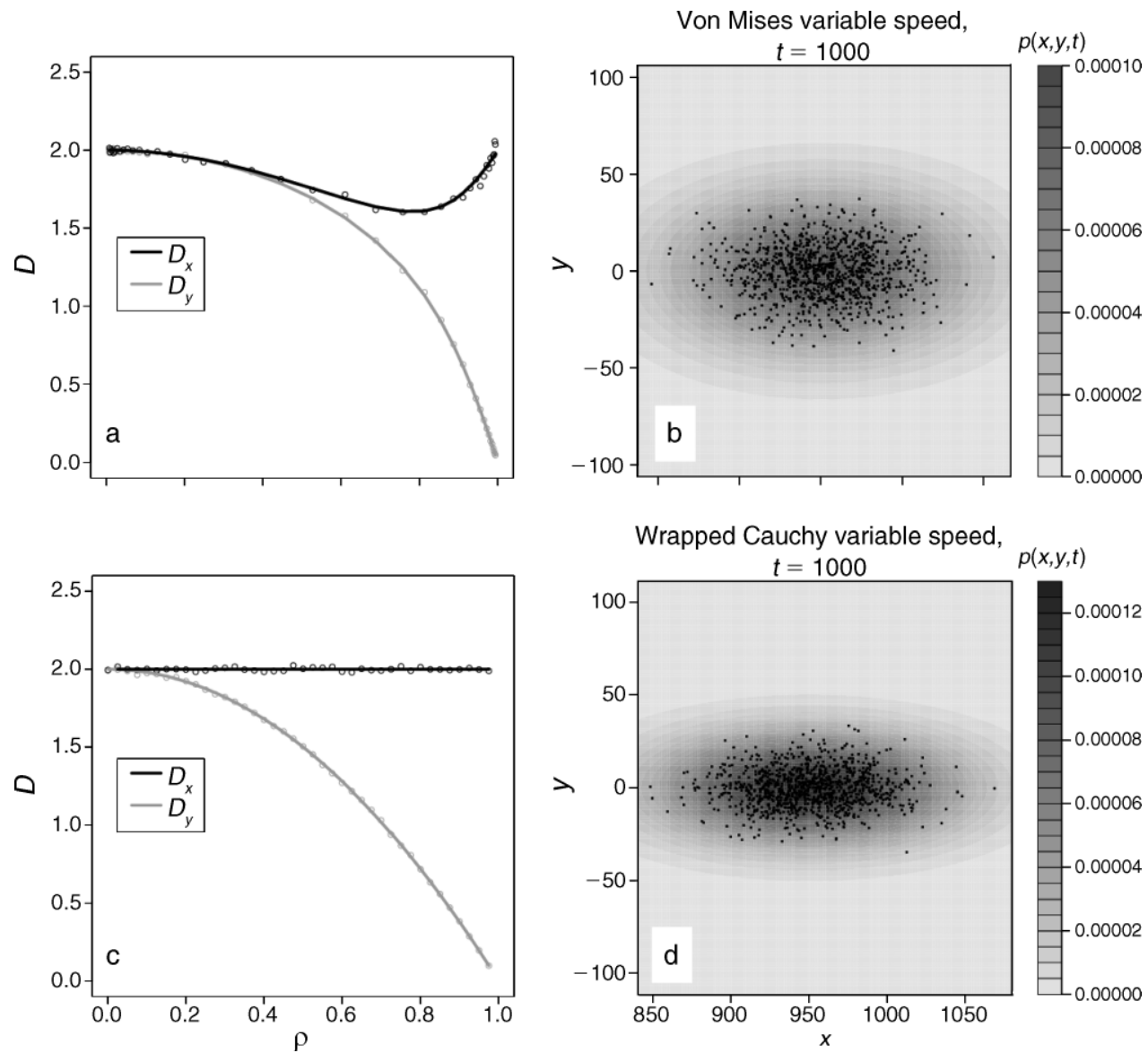

FIG. 3. (a, c) Comparing simulated BRWs to long-time theoretical predictions for (a) the VM distribution and (c) the WC distribution, with variable speed (where the speed is drawn at each step from an exponential distribution with $\sigma_{\delta}^{2}=1$ such that the mean speed is given by $\bar{\delta} / \tau$ ). Theoretical values for the diffusion coefficients in the drift, $D_{x}$, and non-drift, $D_{y}$, directions (rescaled by a factor of $\left.4 \tau /(\bar{\delta})^{2}\right)$ are marked as black $\left(D_{x}\right)$ or gray $\left(D_{y}\right)$ solid lines; simulation results are marked as points. (b, d) Probability density plot calculated from Eq. 4 with $t=1000$ for (b) VM distribution with $\rho=0.95$ and (d) WC distribution with $\rho=0.95$. The end points of 1000 simulated BRWs of 1000 time steps are marked as points. The distance moved in each time step $\tau$ is $\delta$. In the WN distribution, $\sigma$ is the angular variance. In the variable speed case, we assume that the speed at each step is drawn from an exponential distribution with mean speed $\bar{\delta}$ and variance $\sigma_{\delta}^{2}=1$.

diffusion decreases as $\rho$ increases, until $D_{y} \rightarrow 0$ as $\rho \rightarrow 1$ (Fig. 3c). Simulations where the speed is simulated from either an exponential distribution or Gaussian distribution (with $\sigma_{\delta}^{2}=1$ ) give the same results.

\section{Discussion AND CONCLUSIONS}

We have demonstrated how the analytic expressions for the long-time probability density function in twodimensional space and time, $p(x, y, t)$, and the related diffusion coefficients, $D_{x}$ and $D_{y}$, in a BRW are dependent on both the angular distribution used for the choice of direction at each step and whether the random walk process has a fixed or variable speed (where the speed at each step is drawn from an exponential distribution with $\sigma_{\delta}^{2}=1$ ). In particular, we have shown that diffusion about the mean drift position in a BRW is typically anisotropic with $D_{x}<D_{y}$ if the speed is fixed and $D_{y}>D_{x}$ if the speed is variable. The theoretical predictions have been illustrated using individual based simulations.

These results have important consequences for those interested in the movement and dispersal of animal populations. In the simplest derivation of a biased random walk (Codling et al. 2008), the drift (bias) term is assumed to be "weak" in comparison to the diffusive terms and a difference equation is used to derive the drift-diffusion equation. In this case, the interesting anisotropic behavior we have demonstrated is not observed (see discussion in Codling et al. 2008). In many scenarios of animal movement, the drift is not weak relative to the diffusive terms (Benhamou 2006) and hence in such cases our results are likely to be relevant. One of the main assumptions that allows us to make analytic progress with our model is that the system 
is assumed to be at the "long-time limit" such that the spatial distribution is Gaussian. If in our model $\rho \rightarrow 1$, it will take a very long time for this to be true (Hillen 2002). Hence, in scenarios of animal movement where bias is very strong or where the time scale of observation is very short, our results may be less useful (Fig. 2c, d). Similarly, we have not considered short-term correlations in movement due to localized forward persistence in our model (a trait common to many animals). If forward persistence needs to be considered then a biased and correlated random walk (BCRW) can be considered, but this type of model framework is usually far more difficult to deal with analytically than the model presented here (Othmer et al. 1988, Codling and Hill 2005b, Bearon and Grunbaum 2008). However, in most cases, a BCRW considered over a 'long-time limit' is qualitatively similar to a BRW as the correlations in movement direction decay over time (Hillen 2002, Benhamou 2006). Hence our results are also relevant in this context.

The other key assumption in our model is that the random walk is a stationary process in the sense that there is only one mode of behavior throughout the time period we consider. For example, if the random walkers are allowed to change behavior during the walk or if the bias direction changes over time or space (e.g., in directed movement toward a point source such as in Codling et al. 2004 or Benhamou 2006), our results will no longer hold. We have demonstrated how the addition of a variable speed to the model can significantly affected the diffusive behavior, although we only considered a special case of an exponential distribution with $\sigma_{\delta}^{2}=1$. It would be an interesting extension of this study to see how $\sigma_{\delta}^{2}$ and $\rho$ interact in the model for different speed and angular distributions, and for what parameter values we have $D_{x}=D_{y}$. Similarly, we have only demonstrated how analytic expressions can be generated for angular distributions corresponding to movement in a two-dimensional plane. However, in many scenarios (particularly with swimming or flying organisms), it is of interest to model movement in three dimensions and our approach could be developed further to include spherical distributions for the movement direction. This is beyond the scope of our current study but remains an important challenge for future work.

\section{Literature Cited}

Alt, W. 1980. Biased random walk models for chemotaxis and related diffusion approximations. Journal of Mathematical Biology 9:147-177.
Bearon, R. N., and D. Grunbaum. 2008. From individual behaviour to population models: a case study using swimming algae. Journal of Theoretical Biology 251:679697.

Benhamou, S. 2003. Bicoordinate navigation based on nonorthogonal gradient fields. Journal of Theoretical Biology 225:235-239.

Benhamou, S. 2006. Detecting an orientation component in animal paths when the preferred direction is individual dependent. Ecology 87:518-528.

Benhamou, S., and P. Bovet. 1992. Distinguishing between elementary orientation mechanisms by means of path analysis. Animal Behavior 43:371-377.

Berg, H. C. 1983. Random walks in biology. Princeton University Press, Princeton, New Jersey, USA.

Cheung, A., S. Zhang, C. Stricker, and M. V. Srinivasan. 2007. Animal navigation: the difficulty of moving in a straight line. Biological Cybernetics 97:47-61.

Cheung, A., S. Zhang, C. Stricker, and M. V. Srinivasan. 2008. Animal navigation: general properties of directed walks. Biological Cybernetics 99:197-217.

Codling, E. A., and N. A. Hill. 2005a. Sampling rate effects on measurements of correlated and biased random walks. Journal of Theoretical Biology 233:573-588.

Codling, E. A., and N. A. Hill. 2005b. Calculating spatial statistics for velocity jump processes with experimentally observed reorientation parameters. Journal of Mathematical Biology 51:527-556.

Codling, E. A., N. A. Hill, J. W. Pitchford, and S. Simpson. 2004. Random walk models for the movement and recruitment of reef fish larvae. Marine Ecology Progress Series 279: 215-224.

Codling, E. A., M. J. Plank, and S. Benhamou. 2008. Random walk models in biology. Journal of the Royal Society Interface 5:813-834.

Coscoy, S., E. Huguet, and F. Amblard. 2007. Statistical analysis of sets of random walks: how to resolve their generating mechanism. Bulletin of Mathematical Biology 69: 2467-2492.

Hill, N. A., and D. P. Häder. 1997. A biased random walk model for the trajectories of swimming micro-organisms. Journal of Theoretical Biology 186:503-526.

Hillen, T. 2002. Hyperbolic models for chemosensitive movement. Mathematical Models and Methods in Applied Sciences 12:1007-1034.

Mardia, K. V., and P. E. Jupp. 1999. Directional statistics. Wiley, Chichester, UK.

Marsh, L. M., and R. E. Jones. 1988. The form and consequences of random walk movement models. Journal of Theoretical Biology 133:113-131.

Newman, M. E. 2005. Power laws, Pareto distributions and Zipf's law. Contemporary Physics 46:323-351.

Okubo, A., and S. A. Levin. 2001. Diffusion and ecological problems: modern perspectives. Springer-Verlag, Berlin, Germany.

Othmer, H. G., S. R. Dunbar, and W. Alt. 1988. Models of dispersal in biological systems. Journal of Mathematical Biology 26:263-298.

Patlak, C. S. 1953. Random walk with persistence and external bias. Bulletin of Mathematical Biophysics 15:311-338. 